\title{
Economic value of electric vehicle reserve provision in the Nordic countries under driving requirements and charger losses
}

Thingvad, Andreas; Ziras, Charalampos; Marinelli, Mattia

Published in:

Journal of Energy Storage

Link to article, DOI:

10.1016/j.est.2018.12.018

Publication date:

2019

Document Version

Peer reviewed version

Link back to DTU Orbit

Citation (APA):

Thingvad, A., Ziras, C., \& Marinelli, M. (2019). Economic value of electric vehicle reserve provision in the Nordic countries under driving requirements and charger losses. Journal of Energy Storage, 21, 826-834.

https://doi.org/10.1016/j.est.2018.12.018

\section{General rights}

Copyright and moral rights for the publications made accessible in the public portal are retained by the authors and/or other copyright owners and it is a condition of accessing publications that users recognise and abide by the legal requirements associated with these rights.

- Users may download and print one copy of any publication from the public portal for the purpose of private study or research.

- You may not further distribute the material or use it for any profit-making activity or commercial gain

- You may freely distribute the URL identifying the publication in the public portal 


\title{
Economic Value of Electric Vehicle Reserve Provision in the Nordic Countries under Driving Requirements and Charger Losses
}

\author{
Andreas Thingvad, Charalampos Ziras, Mattia Marinelli
}

\begin{abstract}
Electric vehicles can be used for delivering primary frequency control (PFC) and the revenue can compensate for the costs of driving. However, the average system frequency can be biased over the hour, which can lead storage units performing PFC to become either fully charged or depleted. This is also called the energy content of the frequency. Another important role is played by the V2G charger efficiency, which negatively affects the service energy flow. In the paper, the characterisation of the charger and the influence of the losses are detailed. Real frequency and market data are used for calculating the revenue under the Nordic regulatory framework. Earnings are calculated for the best case where the future energy content is known in advance. The results show that, in order to fulfill the service delivery specifications, a crucial role is played by the bid power compared to the size of the energy storage. Recommendations are given in order not to fail regulatory requirements along with considerations on the influence of service provision on the degradation.
\end{abstract}

Index Terms-Ancillary Services, Battery degradation, Electric Vehicles, Frequency Control, Vehicle-to-Grid

\section{NOMENCLATURE}

$f_{t} \quad$ System frequency at time $t$.

$y_{t} \quad$ Normalised power response for PFC on the grid side at time $t$.

$\hat{y}_{t} \quad$ Normalised power response for PFC on the battery side at time $t$.

$P_{t} \quad$ Power response from PFC at time $t$.

$P_{\text {cap }} \quad$ Rated power of converter for both charging and discharging

$N \quad$ The number of measurements of the system frequency per hour.

$n \quad$ The period index during the year

$k \quad$ The number of hours in period $n$

$\omega, h \quad$ Optimisation index; scenario/day index,

hour index.

$K, H \quad$ Optimisation horizon,Number of days and number of hours.

$\Delta t \quad$ The optimisation time step.

$e_{n}^{\text {bias }} \quad$ Normalised energy content of the grid frequency in hour $n$.

$e_{n}^{\text {bat }} \quad$ Normalised energy delivered to the battery in hour $n$.

The work in this paper has been supported by the research projects ACES (EUDP grant nr: EUDP17-I-12499). www.aces-bornholm.eu

The authors are from Center for Electric Power and Energy, Department of Electrical Engineering. DTU - Technical University of Denmark, Roskilde, Denmark. Email: \{athing,chazi,matm\}@elektro.dtu.dk

$S O C_{k, h}$
$\overline{S O C}, \underline{S O C}$
$c_{h}^{\mathrm{E}}$
$c_{h}^{\mathrm{r}}$
$P_{h}^{\mathrm{c}}, P_{h}^{\mathrm{d}}$
$P_{h, \omega}^{\mathrm{c}}, P_{h, \omega}^{\mathrm{d}}$
$P_{h}^{\mathrm{r}}$
$l_{n}^{\text {intra }}$
$l_{n}^{\text {bias }}$
$l_{n}^{\text {all }}$
$E_{h, \omega}$
$Q$
$\eta_{c}, \eta_{d}$
$E_{\text {drive }}$

State of charge of the EV battery in a certain hour and day.

Upper and lower limit of the State of charge.

Electricity price at day-ahead spot market. PFC capacity payment.

Charging and discharging power scheduled day-ahead.

Charging and discharging power traded at the intra-day market one hour before.

Committed power capacity for PFC reserve.

Intra-hourly energy loss at hour $n$.

Hourly bias loss at hour $n$.

Total energy loss at hour $n$.

Scheduled hourly energy flow in a specific hour.

Battery capacity in $\mathrm{kWh}$.

Charging and discharging efficiency.

Daily energy consumption for driving.

\section{INTRODUCTION}

The electrification of the transportation sector is expected to substantially facilitate the use of electric vehicles (EVs). Controlling the charging would make it possible for the EVs to minimise their energy costs through adaptive/predictive charging and for the power grid to achieve multiple operational objectives simultaneously [1]. The use of EVs for providing ancillary services to the power system can be an additional revenue for $\mathrm{EV}$ owners and can assist the integration of larger amounts of renewable sources [2, 3]. A fast response of battery-based energy resources can cover the regulation requirements of significantly larger generating units with slower ramp rates, which are decreasing in numbers, as the generation is transitioning towards renewable production [4].

Any kind of smart integration of EVs with the power system can rely on either bidirectional or unidirectional charging [3, 5]. The unidirectional integration has been defined by the authors in [6] as a power adjustment maintained from a particular moment, for a certain duration, and at a specific location. External bidirectional DC chargers enable the bidirectional integration and can substantially increase the capabilities and profitability of EVs, since they are able to offer power to the system, a concept referred to as Vehicle-to-Grid (V2G) [7, 8]. 
Unidirectional integration involves limiting the power of the internal EV charger with no extra infrastructure, other than the communication and control system [3]. In this case, the EV can still be used for both upward and downward regulation services, as the power can either be decreased or increased. Because of the inexpensive setup and the relatively high losses currently associated with V2G chargers, [9] concludes that only unidirectional services are feasible in practice. Unidirectional integration involves no additional charged energy because the efficiency of the charger does not change significantly when reducing power [10], and since no discharge occurs, there is no additional cost of energy or wear of the battery.

A suitable ancillary service for EVs is primary frequency control (PFC), because it is compensated per power capacity and the energy requirements are relatively small. It is beneficial for EVs because they can have a large power capacity and very fast response, but limited energy capacity [11]. In this service, loads/generators are expected to modify their consumption/production according to the frequency deviation signal in a linear way, as contracted with the Transmission System Operator (TSO) [12].

The TSO will not make contracts with individual EVs because their contribution is negligible, and it would be an excessive burden to trade and control the service of so many units. The minimum bid size of PFC in Denmark is 0.3 MW. There has to be an entity that pools the resources and makes a collective bid, referred to as the aggregator. The value of each EV as a resource for the aggregator depends both on the period length it is connected and available for the grid and how predictable the plug-in and plug-out times are. Ref. [13] finds that by analysing the individual user profiles, the average EV can deliver one hour more of PFC with the same reliability compared to when the plug-in prediction was based on the overall group behaviour. By bidding less than the full capacity, the aggregator of a large group of EVs can make sure that the departure of a few EVs does not compromise the delivery of the service, as the remaining capacity can be used to compensate. The overall charged energy or state of charge (SOC) of a large fleet is also more predictable than the SOC of individual EVs and the aggregator can distribute the charging and discharging between the EVs to align the SOC, so it is only necessary to estimate the average [14].

Modern optimisation methods can be used to schedule and bid in the reserve market and the day ahead spot market, to achieve an optimal economic performance. In [15], the authors calculate the optimal regulation bid in terms of capacity payment with dynamic programming. The purpose of the method is to maximise the value of the regulation and fully charge the EVs in the hours when the spot prices are lowest. The problem in this approach is that it assumes that the positive and negative regulation is equal and results in zero energy exchanged with the grid over time.

If the frequency deviation is unbiased, i.e. its integral over a time period is zero, then the overall energy exchange between the EV and the grid would be very small. However, frequency deviations can be significantly biased in consecutive hours, which could lead to relatively large energy exchanges. The energy content of the frequency is not a problem for a thermal power plant, but for a storage unit like an EV with a limited battery capacity, it is very likely that the batteries will be fully charged or depleted within the reserve provision period; in that case, the aggregator will not be able to provide the committed reserves. Ref. [9] takes the energy content into account in the scheduling phase when making an optimisation with dynamic programming, but only for unidirectional PFC and only when the same reserve capacity is delivered in all the hours. Ref. [16] is scheduling delivery to the Automatic Generation Control to the California Independent System Operator, which is a secondary control that is high-pass filtered to have a zero energy content over an hour, and has a performance-based compensation.

In Europe, PFC has a capacity-based payment, as it is contracted to guarantee delivery. In ref. [17], PFC in Regional Group Continental Europe (RG-CE) is analysed and the variance of frequency is causing an uncertainty for the State of Charge (SOC) of storage units delivering the service. The revenue is found to be increased by up to $25 \%$ by co-optimising the day-ahead scheduling of PFC and self consumption, compared to only delivering PFC. In order to have an optimisation time step like the spot market, [17], calculates the average normalised frequency deviation over the hour. In order to get a linear problem, the charging and discharging efficiency is added to the normalised frequency before the hourly deviation is calculated. Both methods are applied in the present manuscript, but more analysis is given to the consequences of these simplifications.

Ref. [18] shows that when not committing the full power capacity of a battery for PFC, the remaining power can be used for constraining the SOC by changing the operating point during operation. Ref. [18] finds that a wide range of allowed SOC is preferable to minimise the use of correcting power, because some energy content cancels out the previous.

The average daily driving distance of cars with internal combustion engines in Denmark is $45 \mathrm{~km} / \mathrm{day}$, which considering an average consumption of $5 \mathrm{~km} / \mathrm{kWh}$ results in a consumption of $9 \mathrm{kWh} /$ day. As the battery capacity of the new EV models increases, the daily energy use becomes a smaller part of the total capacity. The EV therefore does not have to be fully charged at the end of service provision, as for instance assumed in [9, 15]. It is possible to provide PFC until the EV is needed for driving without allocating time for pure charging, when it is not required to have it fully charged.

In [19], a data-driven model based on historical Automatic Generation Control activation data is presented, modelling the uncertainty of SOC as a function of the reserve capacity and provision time. In ref. [11], the energy content of the frequency in the Nordic grid is modelled as an increasing confidence interval as a function of the service provision capacity and duration. The uncertainty can only be modelled as an interval when the same PFC capacity is delivered every hour. This way of modelling the PFC energy content is only accurate when the reserve capacity is constant during the whole period, because the energy content of a certain hour is correlated with the content of previous hours. This relationship is used by [20] to predict the future energy content based on the values of the 
last two hours. By using scenarios it is possible to take the behaviour of the frequency into account without having the same reserve capacity in all hours. The energy losses due to charger efficiency are found to converge to an average value, that can be approximated with a constant efficiency.

In the present manuscript, the real energy content of the frequency in the periods where the service is delivered, is used as scenarios in a stochastic optimisation. This gives a more accurate modelling of the behaviour of the energy content. The method of using scenarios to make a robust schedule is also suggested by [21], where 100 scenarios are generated from a Gaussian distribution per period. This does not capture the correlation between the hours and specific time behaviour, which can be exploited by using the real frequency for the scenarios.

An additional challenge is introduced by the efficiency of the chargers, which causes energy losses to the EV batteries, and which must be also accounted for, when bidding in the power markets. In ref. [11] the energy losses due to charger efficiency are found to converge to an average value, that can be approximated with a constant efficiency. The authors of [22] have made an overview of literature stating that losses in the charger are often neglected or considered very low. While the charger efficiency presented in this paper does not make the service provision technically impossible, it does reduce the profit dramatically and completely cancels out the profit if the electricity price is including tariffs and taxes. This paper will present a comprehensive characterisation of the charger efficiency to make the economical analysis more realistic.

The main contributions of this paper are the following:

- Realistic earnings based on historical market data and charger efficiency.

- Analysis of accuracy of representing the charger losses on an hourly basis.

- A method for optimal scheduling taking energy constraints and frequency behaviour into account.

- Considerations on the consequences on the battery degradation because of intense service provision.

The remainder of the paper is structured as follows. Section II presents an overview of the grid code regarding provision of PFC in the Nordic countries, and to which extent the EVs can deliver the service by different charging methods. The section also presents measurements of the efficiency of a commercial V2G charger. Section III contains a description of the behaviour of the frequency and how it affects the SOC of the EV during PFC provision. It also presents an analysis how the frequency affects the charger losses, caused by continuous charging and discharging and how this can be estimated on an hourly resolution. Finally, the section presents the optimisation model and cost function. Section IV presents the result of the optimisation, which are validated based on full resolution time series simulations, and the charger loss estimation errors are calculated. A discussion on the influence of battery degradation concludes the section. Section $\mathrm{V}$ concludes the paper.

\section{BACKGROUND}

\section{A. Frequency normal operation reserve}

The Danish power system is part of two synchronous zones: Western Denmark (DK-1) is a part of RG-CE, and Eastern Denmark (DK-2) is a part of the Regional Group Nordic (RG-N). DK-2 is the focus of this paper. In both parts PFC reserve is paid per available power capacity, $P_{\text {cap }}$, for each hour, independent of how often and how much the reserve is activated, and the price is determined by the market. Fig. 1 shows the average capacity payment in DK-1 and DK-2 during the day.

The average capacity payment for the last two years is 21.4 EUR/MW per hour in DK-1 and 24.1 EUR/MW per hour in DK-2, but the capacity payment in DK-2 is close to three times higher than in DK-1 during the night, where the EVs are often available for grid services.
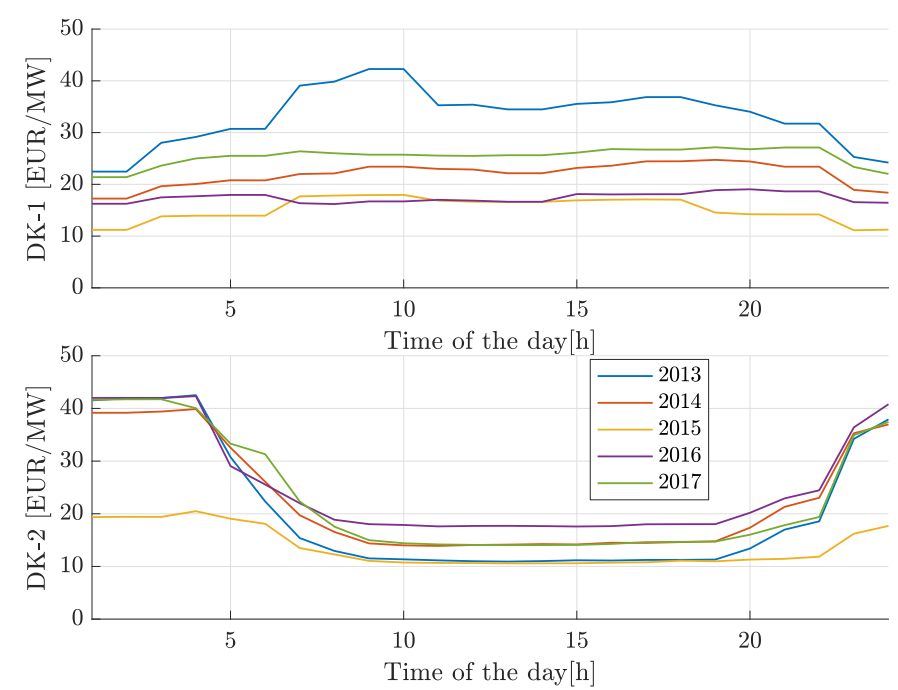

Fig. 1. Average hourly capacity payment in DK-1 and DK-2

The prices in DK-2 were lower in 2015 because it was a year with extraordinary high amount of rain in the Nordic region, which caused the hydro power plants to deliver more reserve than usual and thereby caused the prices to decrease. Apart from the hourly variability of the regulation prices, a seasonal variability is also observed, with prices in summer generally being $30 \%$ higher than in winter. This is caused by the large number of combined heat and power plants in the system, that are only delivering services in the winter, when they also are delivering heat. The PFC service in DK-2 is called Frequency Normal-operation Reserve (FNR), and has the average seasonal prices shown in Table I

TABLE I

AVERAGE SEASONAL FNR CAPACITY PRICES FOR 2017

\begin{tabular}{c|c|c|c|c} 
Season & Winter & Spring & Summer & Autum \\
\hline EUR/MW & 22.13 & 22.46 & 29.14 & 21.84
\end{tabular}

The Transmission System Operators (TSOs) in RG-N are jointly responsible for procuring $600 \mathrm{MW}$ of FNR reserves, proportional to each TSO's share of the production. The 
service is bought on market terms one or two days ahead with a minimum bid size of $0.3 \mathrm{MW}$. FNR is a symmetrical service, which requires the provider to offer the same power capacity for upwards and downwards regulation. Frequency reserves must be provided linearly, with full activation for deviations of $\pm 100 \mathrm{mHz}$, without a deadband. For a frequency value $f_{t}$ at time $t$, the normalised response $y_{t}$ is calculated as

$$
y_{t}=\left\{\begin{aligned}
-1, & \text { if } \quad f_{t}<49.9 \mathrm{~Hz} \\
\left(f_{t}-50\right) / 0.1, & \text { if } 49.9 \mathrm{~Hz} \leq f_{t} \leq 50.1 \mathrm{~Hz} \\
1, & \text { if } \quad f_{t}>50.1 \mathrm{~Hz}
\end{aligned}\right.
$$

The power required by the service provider at time $t$ is calculated as

$$
P_{t}=P_{\text {cap }} \cdot y_{t}
$$

\section{B. FNR provision with electric vehicles}

FNR can be provided with series-produced EVs in two ways; by modulating the unidirectional power flow using the on-board $3.7 \mathrm{~kW}$ charger seen on the right of Fig. 2, or with an external bidirectional charger seen on the left of the figure. Fig. 2 shows the different charging options for Nissan LEAF, which is considered in this study.

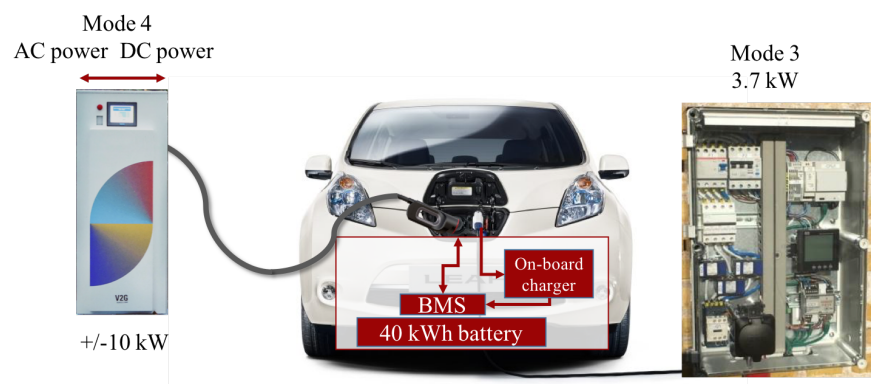

Fig. 2. Physical setup - Nissan LEAF with different charging options

1) Potential value of unidirectional FNR: The lowest charging value allowed by the IEC 61851 and SAE J1772 standards is $6 \mathrm{~A}$. The maximum possible reserve, when performing unidirectional FNR with a single $\mathrm{EV}$, is obtained by setting the charging set point to $11 \mathrm{~A}$. In that case, the current can be modulated with $\pm 5 \mathrm{~A}$ in a range of $6-16 \mathrm{~A}$, giving a FNR capacity of $\pm 1.15 \mathrm{~kW}$ [3].

Providing FNR with the internal charger involves low installation cost because all power conversion occurs inside the EV. The disadvantage is that the service can only be provided until the EV is fully charged, which on average would be after receiving $9 \mathrm{kWh}(45 \mathrm{~km})$ of energy. Several series-produced EVs have been found to have an efficiency of the on-board charger of around $90 \%$. Given the daily driving consumption, the efficiency of the charger and a charge cycle with an average of $11 \mathrm{~A}(2.5 \mathrm{~kW})$, the EV would be fully charged in four hours [10]. Using an EV with a higher charging power or energy capacity would not increase the revenue, since the limiting factor is the energy demand from driving. The average charging time is four hours, which means that in the days where the frequency on average is too high, the EV would charge with higher power and be fully charged sooner. Three hours of service would therefore be a more realistic estimate.
2) Potential value of bidirectional FNR: Providing bidirectional FNR lifts this time limitation, as the EV over time is expected to deliver the same amount of energy to the grid as it receives, giving a zero energy balance. It is therefore only the daily number of grid-connected hours and the power capacity of the power converter that determines the potential capacity payment. Assuming that the EV is parked from 16:00 in the evening to 07:00 in the morning, it would be available for 15 hours to provide grid services. The energy company ENEL has produced a bidirectional charger, seen on the left in Fig. 2, with a capacity of $\pm 10 \mathrm{~kW}$ that via the CHAdeMO DC connection can be used to perform FNR with all series-produced EVs using that standard.

During a pilot project with the Danish TSO and DTU, a commercial operation of EVs providing FNR in Denmark with 40 of the $\pm 10 \mathrm{~kW} \mathrm{V2G}$ chargers and EVs. The used EVs are the Nissan e-NV200 with a battery capacity of $24 \mathrm{kWh}$ and Nissan LEAF with $30 \mathrm{kWh}$ capacity. The EVs do not allow discharging below $35 \%$ and cannot charge with full power above $90 \%$, which means that the available capacity is 13.2 and $16.5 \mathrm{kWh}$ respectively. With a SOC in the middle of the range, the EVs could both deliver and receive half of the amount, and could be fully charged or depleted within one hour. To handle this problem, the aggregator is offsetting the power dynamically as a function of the SOC, to avoid being fully charged or depleted. This is done even though the full charger capacity has been committed for FNR, which means it does not live up to the regulative requirements in the RG-CE or RG-N [23]. The aggregator reports a yearly revenue of $1000-1400$ EUR per EV, based on an average plug-in time of 19 hours per day [24]. The experimental results have shown that such an offset on a daily basis would get up to $5 \mathrm{~kW}$. This means that in order to deliver the service with a capacity of $\pm 10 \mathrm{~kW}$ it would be necessary to have a $\pm 15 \mathrm{~kW}$ charger [23].

\section{Characterising the charger efficiency}

Since large amounts of energy are exchanged with the grid during FNR provision, the efficiency of the power converter has a high impact on the energy consumption. This paper presents a characterisation of the $\mathrm{V} 2 \mathrm{G}$ charger that can make the foundation for further usage with new control algorithms. The characterisation is made by charging and discharging with all possible power levels with a Nissan LEAF $30 \mathrm{kWh}$ battery at the whole range of SOC. The power is set on the DC side with a granularity of $1 \mathrm{~A}$, giving a step size of about 0.4 $\mathrm{kW}$. The steady-state powers on both sides of the charger are averaged over one minute and divided to calculate the efficiency. The charging efficiency is found as $\eta_{\mathrm{c}}=P_{D C} / P_{A C}$ and the discharging efficiency as $\eta_{\mathrm{d}}=P_{A C} / P_{D C}$. The whole range of power values are investigated for different SOC as shown in Fig. 3 .

The extremely low efficiency at power ratings below \pm 3 $\mathrm{kW}$ is caused by the standby loss not being a function of the power. The voltage level on the DC side is a function of the SOC but does not have an effect on the efficiency. Based on measurements of the energy losses in the V2G charger 


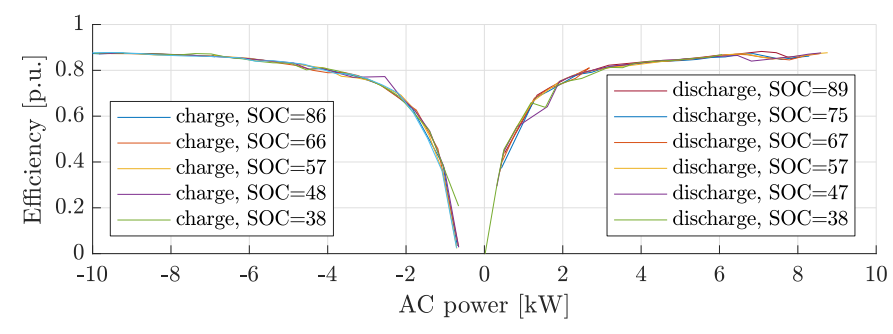

Fig. 3. Charger efficiency during charge and discharge cycles at different SOC levels

performing FNR during 24 hours, it was found that the energy loss per hour converges to a certain value that corresponds to an average efficiency of $80 \%$ [11]. The charging and discharging efficiency is chosen to be equal $\eta_{\mathrm{c}}=\eta_{\mathrm{d}}=0.8$, which will be used in the rest of the paper.

\section{DisPatching STRATEGY}

\section{A. Frequency Energy Content Model}

The analysis has been based on a data set consisting of one year of system frequency measurements from RG-N with a sample rate of 10 seconds. The integration of frequency deviations of a given hour $n$, referred to as the energy content or energy bias of that hour, is denoted by $e_{n}^{\text {bias }}$. For a sample rate of $t_{\mathrm{s}}=10 \mathrm{~s}$, the number of samples per hour is equal to $N=360$. The per unit energy content (normalized per $\mathrm{kW}$ of regulation capacity), is given by

$$
e_{n}^{\text {bias }}=\frac{1}{N} \sum_{t=N \cdot(n-1)+1}^{n \cdot N} y_{t} \cdot t_{\mathrm{s}}
$$

The distribution of $e_{n}^{\text {bias }}$ for every hour of the year is Gaussian with zero mean.

Since frequency is strictly related to the balance of a power system, it is based on a multitude of power inputs and outputs from generators and consumers. While some power injections are controlled by prime movers, most of the remaining are randomly distributed. As a consequence, frequency patterns will tend to follow a normal distribution. The integral of frequency deviations is kept close to zero over time due to the applied time control. Thus, the energy bias also tends to be zero over time. However, the variance of the energy bias of $k$ consecutive hours does not increase linearly with $k$, because of the correlation of $e_{n}^{\text {bias }}$ for different hours. We introduce $e_{n}^{\text {bias, } k}$ to denote the energy bias of the period starting $k$ hours before $n$ and ending at hour $n$.

$$
e_{n}^{\text {bias }, k}=\frac{1}{N} \sum_{t=N \cdot(n-k)+1}^{n \cdot N} y_{t} \cdot t_{\mathrm{s}}, \quad n>k .
$$

It is now possible to calculate a confidence interval for the energy content which the service provider could be asked to deliver or receive with a certain probability. In Fig. 4, the $99.5^{\text {th }}$ percentile of the energy content is shown, as a function of $k$ [11]. Note that the distribution of $e_{n}^{\text {bias }, k}$ for each $k$ is symmetrical. Therefore, the $0.5^{\text {th }}$ percentiles have the same values with a negative sign.

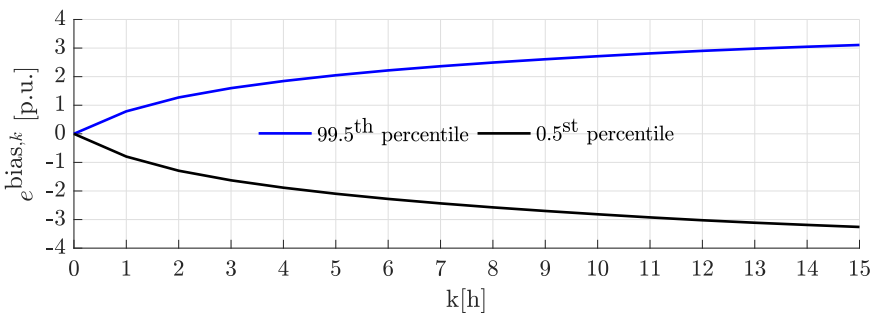

Fig. 4. $99.5^{\text {th }}$ percentile of the distribution of $e^{\text {bias, } k}$ as a function of $k$.

The percentiles of the energy content are calculated per PFC capacity in $\mathrm{kW}$ and scales linearly with the reserve capacity. PFC provision with $\pm 10 \mathrm{~kW}$ for 15 hours could result in receiving or delivering up to $31 \mathrm{kWh}$ of energy, requiring an available battery capacity above $62 \mathrm{kWh}$. Using the 2018 Nissan LEAF with a $40 \mathrm{kWh}$ battery and $35-90 \%$ of its SOC available, would mean that PFC could only be delivered for 15 hours with a capacity of $\pm 3.5 \mathrm{~kW}$. The limitation of using this description is that it holds only when the reserve capacity is constant over $k$ hours. Short term frequency deviations have a limited effect on the SOC, but Fig. 5 shows the normalised energy content every hour of 2016 and it is seen that there is also a pattern of the hourly energy content and that there are periods of the day where the EVs are expected to receive energy, and others where it is expected to deliver energy to the grid. It is possible to extract more knowledge of the frequency behaviour by defining the specific time of the day the service is provided, and which is the reason for using the energy content from Fig. 5 as scenarios in a stochastic optimisation.

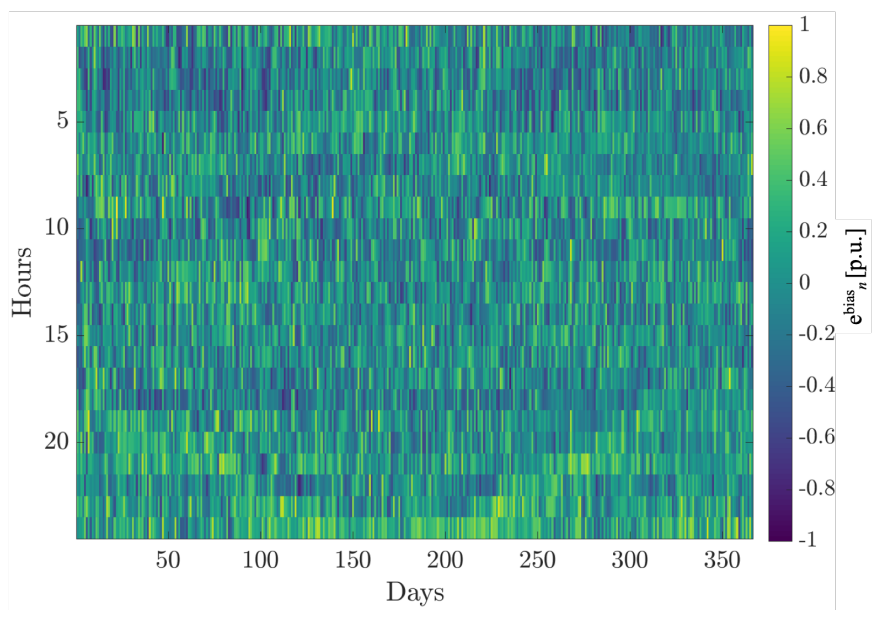

Fig. 5. The values of $e_{n}^{\text {bias }}$ of each hour of the year 2016 .

Ref [11] showed that the short term frequency deviations are following the sunrise and sunset, which are also the times when there are larger changes in the power consumption. It can be seen that the frequency also on a hourly time scale has this behaviour, as it is often too high or too low for an extended period of time. It should also be noted how one hour of over frequency often is followed by one hour of under frequency. 


\section{B. Bias and Intra-hourly losses}

Continuously charging and discharging during reserve provision will cause a loss of energy. The losses can be decomposed into two parts. The first part is the bias loss, $l^{\text {bias }}$, which is caused by the positive or negative frequency bias and is given by Eq. 5 .

$$
l_{n}^{\text {bias }}= \begin{cases}e_{n}^{\text {bias }}\left(1-\eta_{\mathrm{c}}\right) & \text { if } e_{n}^{\text {bias }} \geq 0 \\ e_{n}^{\text {bias }}\left(\frac{1}{\eta_{\mathrm{d}}}-1\right) & \text { if } e_{n}^{\text {bias }}<0 .\end{cases}
$$

The overall losses will be equal to $l_{n}^{\text {bias }}$ only if the EVs are only charging or discharging during this period. If they are alternating between charging and discharging, which is the most common case when FNR is provided, additional losses occur. The remaining losses can be quantified by applying the effect of the efficiency to the full frequency time series. The normalised power measured on the battery side of the charger is denoted by $\hat{y}_{t}$, and the hourly energy received or delivered by the battery is denoted by $e_{n}^{\text {bat }}$.

$$
\begin{gathered}
\hat{y}_{t}= \begin{cases}y_{t} \eta_{\mathrm{c}}, & \text { if } y_{t} \geq 0 \\
y_{t} \frac{1}{\eta_{\mathrm{d}}}, & \text { if } y_{t}<0\end{cases} \\
e_{n}^{\mathrm{bat}}=\frac{1}{N} \sum_{t=N \cdot(n-1)+1}^{n \cdot N} \hat{y}_{t} \cdot t_{\mathrm{s}}
\end{gathered}
$$

The difference between the received energy and the pure energy content is the total loss, $l_{n}^{\text {all }}=\left|e_{n}^{\text {bias }}-e_{n}^{\text {bat }}\right|$. After accounting for the bias loss, the remaining losses occur every time power changes direction. These losses are the second loss component and are referred to as intra-hourly losses, $l_{n}^{\text {intra }}$. The intra-hourly loss is the remaining loss after subtracting the bias loss, $l_{n}^{\text {intra }}=l_{n}^{\text {all }}-l_{n}^{\text {bias }}$, and is the energy lost in the charger because of continuous changing between charging and discharging. Note that while the bias losses are proportional to the reserve capacity, the intra-hourly losses depend on the power reference as well. By power reference we refer to the power set-point when no reserve is provided. Indeed, a high power reference will result in the EV mainly charging and rarely changing to discharging mode; this will happen only when frequency is very low. Fig. 6 shows the bias loss, the intra-hourly loss and the total loss of every hour of 2016 against the energy content of the specific hour and with a zero power reference. It shows that the bias loss is proportional to the energy content and it accounts, on average, for two thirds of the total loss with $\bar{l}^{\text {bias }}=0.0571$ p.u.. $l_{n}^{\text {intra }}$ is larger when the energy content is low, as in those cases power most often changes direction and on average is equal to $\bar{l}^{\text {intra }}=0.024$ p.u.. As the energy content increases in absolute value for a given hour, frequency is predominantly positive or negative throughout this period and power rarely changes direction, resulting in very low $l^{\text {intra }}$ losses.

The intra-hourly loss is different for every $e_{n}^{\text {bias }}$ level, depending on the exact evolution of frequency, but scales linearly with the reserve capacity, $P_{h}^{\mathrm{r}}$, if the set point is zero. The dependency of the operating point on the average intra-hourly loss is shown in Fig. 7. It is shown that the
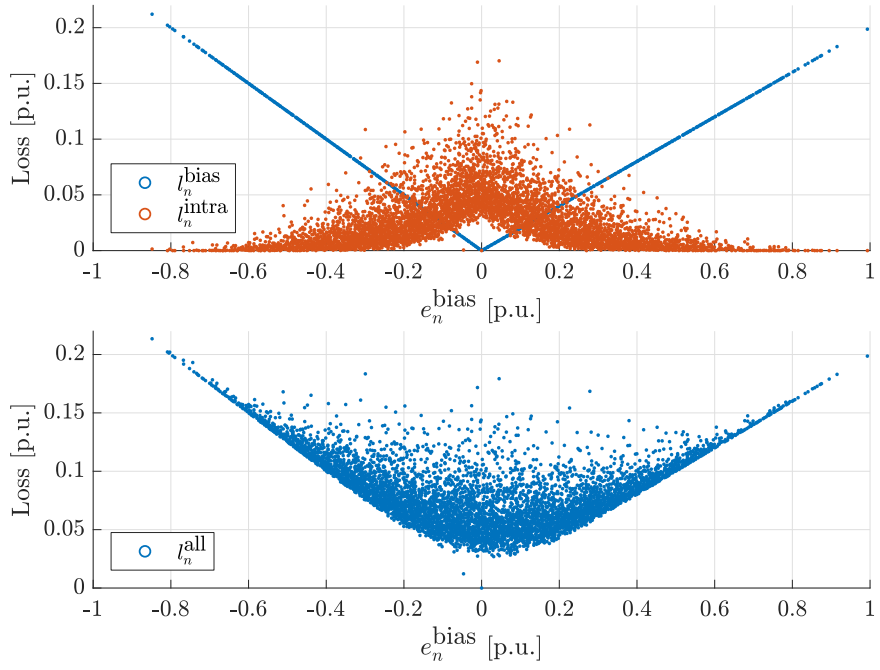

Fig. 6. Hourly bias loss, intra-hourly loss and total loss of every hour of 2016 vs. the energy content in that hour, with $P_{h}^{\mathrm{r}}=1 \mathrm{~kW}$ and $P_{h}^{\mathrm{c}}=0$.

intra-hourly loss is reduced to zero when the charging power is larger than the reserve capacity, as it will never discharge.

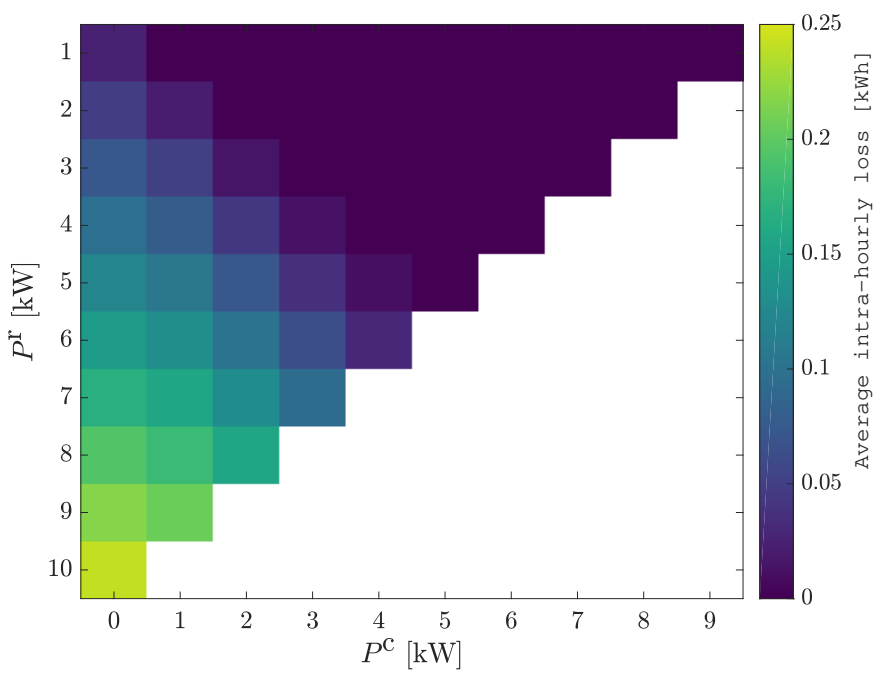

Fig. 7. Average intra-hourly loss. White area represent the combinations larger than the power capacity of the charger.

\section{Linear model of energy flow}

The FNR regulation capacity, $P_{h}^{\mathrm{r}}$, and the day ahead schedules ( $P_{h}^{\mathrm{c}}$ for charging and $P_{h}^{\mathrm{d}}$ discharging) are traded the day before, and are therefore the same for all scenarios. If less than the full power capacity is committed day-ahead, then the remaining power can be used in the individual scenarios for buying or selling additional energy at the intra-day market. It was found that because of the large variance in the energy content, it is never economical to schedule charging at the day-ahead market, as it limits the flexibility for providing reserve. This is the case, even when assuming that the price on the intra-day market is twice as high as on the spot market; all of the energy needed to correct the SOC is bought at the intra-day market. The index $\omega$ is therefore added to $P_{h, \omega}^{\mathrm{c}}$ 
and $P_{h, \omega}^{\mathrm{d}}$ to indicate the per scenario values for the intra-day power schedules. For the scenarios the frequency deviations of each hour from 16:00 to 07:00 the following day were used. For each hour $h$ of a scenario $\omega$, the normalized energy content including all losses, $e_{h, \omega}^{\text {bat }}$, was calculated, considering a charger efficiency of $\eta_{c}=\eta_{d}=0.8 . e_{h, \omega}^{\text {bat }}$ represents the energy flowing in/out of the battery at hour $h$ and for scenario $\omega$, normalized per reserve capacity. This is the real energy content experienced by the battery $e_{h, \omega}^{\text {bat }}$ including all losses $l_{h, \omega}^{\text {all }}$ when the power reference is zero.

As already shown, the intra-hourly losses are a function of both the reserve capacity and the power setpoint (see Fig. 7). In the case where the power setpoint is different from zero, the total intra-hourly losses are reduced. This effect is not taken into account when $e_{h, \omega}^{\text {bat }}$ is scaled with the reserve capacity $P_{h}^{\mathrm{r}}$. This allows us to use a linear formulation to express the total losses. The accuracy of this approach will be investigated in the following section.

The total energy exchange due to reserve provision is calculated by multiplying $e_{h, \omega}^{\text {bat }}$ with $P_{h}^{\mathrm{r}}$. We must note again that this calculation is exact only for zero intra-day power setpoints, as explained earlier. The SOC of the battery $S O C_{h, \omega}$ in scenario $\omega$ can then be calculated as

$$
S O C_{h, \omega}=S O C_{h-1, \omega}+\left(P_{h, \omega}^{\mathrm{c}} \eta_{\mathrm{c}}+e_{h, \omega}^{\mathrm{bat}} P_{h}^{\mathrm{r}}-P_{h, \omega}^{\mathrm{d}} \frac{1}{\eta_{\mathrm{d}}}\right) \frac{\Delta T}{Q} .
$$

The variable $S O C_{h, \omega}$ is in p.u. and the value at the next time step is calculated by dividing the added energy with the battery capacity, $Q$, which is chosen to be equal to $40 \mathrm{kWh}$ as the 2018 Nissan LEAF.

\section{Case description}

The optimisation is based on the average capacity payment shown in Fig. 1 for 2017, and it is assumed that a bid at this price is accepted. In Denmark, the retail price is on average $0.32 \mathrm{EUR} / \mathrm{kWh}$, in which the price for the energy accounts for approximately $12 \%$. However, for the analysed case the mid-sized industrial customer price will be considered. The price does not include VAT and other taxes and it is on average $0.08 \mathrm{EUR} / \mathrm{kWh}[25]$. The reason for choosing this price is that the service is delivered by a commercial aggregator, rather than a residential customer.

It is assumed that the average SOC of the EV fleet can be estimated and is equal to $50 \%$ at the time of arrival. It is assumed that the EV drives $45 \mathrm{~km}$ and consumes $9 \mathrm{kWh}$ per day, which should be compensated while it is connected to the charger so it should leave with a SOC minimum $22.5 \%$ higher than it arrives, giving $72.5 \%$. It is also assumed that the plug-in time is known in advance, as is the case in the ACES project, where the plug-in time follows the working hours of the municipality who uses the fleet [26]. The problem of defining the availability is simplified by assuming that all EVs are at the disposal of the aggregator between 16:00 in the afternoon and 07:00 the following morning, at which time the SOC must be minimum $72.5 \%$. The setup is exactly the same as the real practical implementation in the ACES project, where 20 Nissan EVs, located on Bornholm, are used to provide FNR with the ENEL chargers for 15 hours per work day.

\section{E. Problem formulation}

The optimisation uses a set of 365 days as scenarios, each containing the energy content of the relevant 15 hour period. The objective function contains the cost of energy for charging, the revenue for discharging and the reserve capacity payment, as it is seen from the aggregator's perspective. The costs are positive and the revenue is negative so the objective function should be minimised.

$$
\min _{S O C, P^{\mathrm{c}}, P^{\mathrm{d}}, P^{\mathrm{r}}} \sum_{\omega} \sum_{h=1}^{15} c_{h}^{\mathrm{E}} P_{h, \omega}^{\mathrm{c}}-c_{h}^{\mathrm{E}} P_{h, \omega}^{\mathrm{d}}-c_{h}^{\mathrm{r}} P_{h}^{\mathrm{r}}
$$

subject to the following constraints, applied for $\omega=$ $1, \ldots, 365$ and for $h=1, \ldots, 15$

$$
\begin{gathered}
P_{h, \omega}^{\mathrm{c}}+P_{h, \omega}^{\mathrm{d}}+P_{h}^{\mathrm{r}} \leq P_{\max } \\
P_{h, \omega}^{\mathrm{c}} \geq 0, P_{h, \omega}^{\mathrm{d}} \geq 0, P_{h}^{\mathrm{r}} \geq 0 \\
S O C_{h+1, \omega}=S O C_{h, \omega}+\left[P_{h, \omega}^{\mathrm{c}} \eta_{\mathrm{c}}+e_{h, \omega}^{\mathrm{bat}} P_{h}^{\mathrm{r}}-P_{h, \omega}^{\mathrm{d}} \frac{1}{\eta_{\mathrm{d}}}\right] \frac{\Delta T}{Q} \\
\underline{S O C} \leq S O C_{h, \omega} \leq \overline{S O C}
\end{gathered}
$$

where $\underline{S O C}=0.35$ and $\overline{S O C}=0.9$ the minimum and maximum SOC values respectively. The initial and final SOC conditions $S O C_{1, \omega}=0.5, S O C_{15, \omega} \geq 0.725$ are the same for all scenarios.

\section{RESUlts}

In this section, the results of several investigations will be presented. In subsection IV-A, the linear approximation model regarding the charging/discharging losses is verified by comparing the actual and the real losses for each scenario of the stochastic optimization. In subsection IV-B, the optimization results are validated for a set of out of sample scenarios (i.e. scenarios not used in the uncertainty set), and using the real losses during service provision.

For the optimisation problems the Yalmip environment in Matlab is used, along with the Gurobi solver [27].

\section{A. Validation of the linear approximation model of losses}

A number of 365 scenarios, corresponding to year 2016, along with the average FNR price for 2016 were used for the stochastic optimization, and the result is seen in Fig. 8

The lines of the top graph show the evolution of the SOC for every realization of 2016. The reserve schedule, which is always the same, is seen in the second graph of Fig. 8 These results are obtained by assuming intra-day losses which correspond to a zero power setpoint. However, this is not the case for every realization of frequency. In order to calculate the approximation errors, losses were evaluated using the full resolution time-series of frequency, and for the realized 

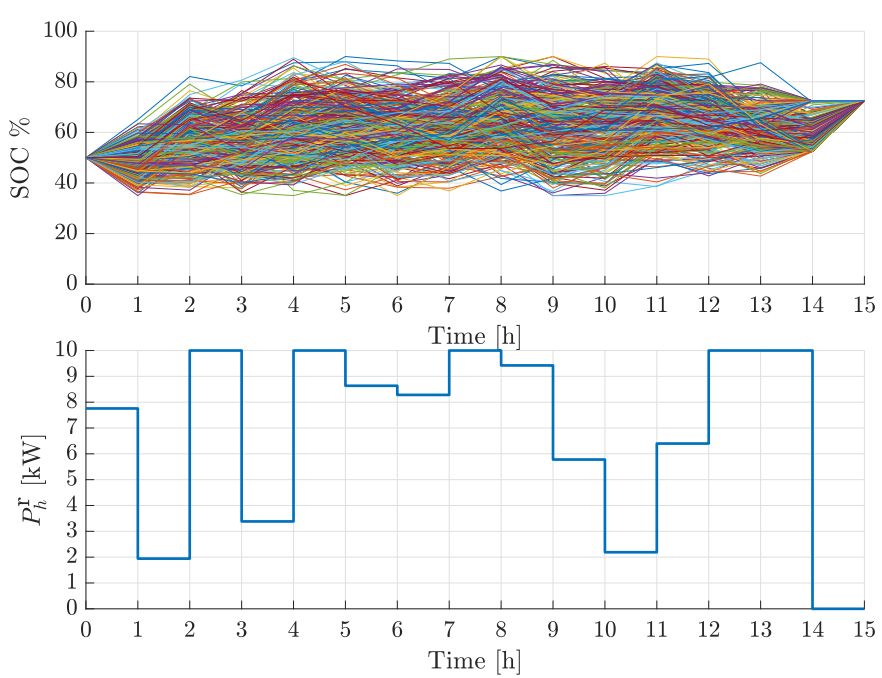

Fig. 8. Top: SOC for each of the 365 frequency realizations of 2016 using the linear approximation. The 15 hour periods take place daily at hour 16:00-07:00. Bottom: Reserve schedule in all scenarios, calculated for 2016 prices.

intra-day rescheduled power setpoints for each scenario. The evolution of the actual SOC (reflecting the real losses) for each realization is seen in Fig. 9

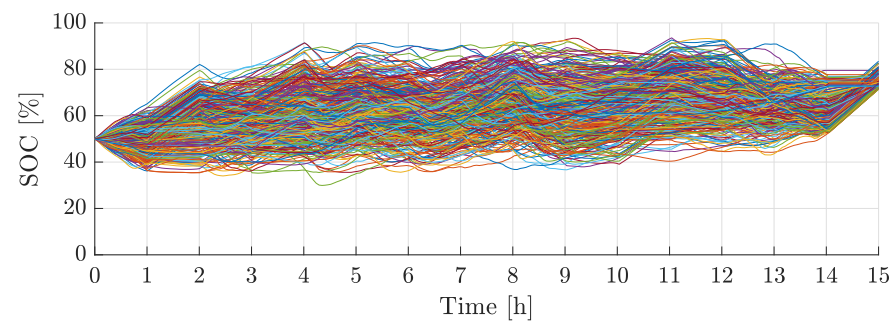

Fig. 9. Simulation with 10-second resolution frequency time series of 2016 . The 15 hour periods take place daily at hour 16:00-07:00

The simulation results are obtained by using an average, constant efficiency of $80 \%$. As shown previously, efficiency depends on each EV's power setpoint (see Fig. 3). However, an aggregation of EVs can achieve higher average efficiencies regardless of the power setpoints, by applying appropriate control methods [28]. As shown previously, even for a constant considered efficiency, the intra-hourly losses depend on the power setpoint, and in these simulations this effect is taken into account. The approximation error can be found by subtracting the SOC calculated based on the full resolution time series (Fig. 9p from the one calculated based on the hourly schedule (Fig. 88). In Fig. 10, the distributions of the accumulated approximation errors in the 365 scenarios are shown, for each hour.

According to the optimisation results using the losses approximation model, EVs receive $9 \mathrm{kWh}$ every day, whereas the average energy according to the full resolution time series (which is the real result) is $10 \mathrm{kWh}$. This is a result of the small overestimate of losses, which on average accumulate to $1 \mathrm{kWh}$ over 15 hours, as seen in Fig. 10 Additionally, in 99\% of the cases, the losses overestimation amounts to less than 3

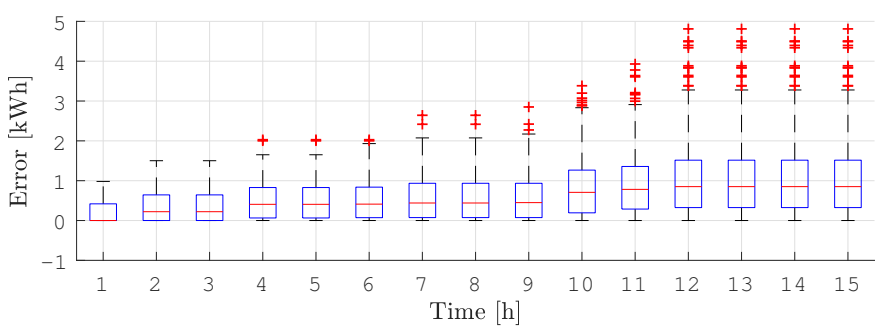

Fig. 10. Distribution of the accumulated approximation error of intra-hourly losses. The box shows $25-50-75$ percentiles, - shows the $1^{\text {st }}$ and $99^{\text {th }}$ percentile and + shows the outliers.

$\mathrm{kWh}$. Compared to the EVs energy capacity of $40 \mathrm{kWh}$, the approximation error are rather small.

\section{B. Evaluation for out of sample frequency realizations}

In this set of simulations, the same frequency scenarios from 2016 are used to obtain the FNR reserve schedule. Contrary to before, the realizations of frequency correspond to 2017, and thus are not contained on the uncertainty set. The optimal values of $P_{h, \omega}^{\mathrm{c}}$ and $P_{h, \omega}^{\mathrm{d}}$ are calculated based on the energy content of each realization of 2017 , by using the deterministic counterpart of the stochastic optimization problem. The optimisation is in this case allowed to violate the energy constraints with a high cost to make sure that the problem is feasible. A simulation is made with the same capacity in every hour, which is the average capacity, $\overline{P_{h}^{\mathrm{r}}}= \pm 6.9 \mathrm{~kW}$.

The real losses are calculated in the same way as in subsection IV-A, to obtain the actual SOC evolution for each realization (similar to Fig. 9). In Fig. 11 the minimum, maximum, $1^{\text {st }}$ and $99^{\text {th }}$ percentiles of the realized SOCs for every hour when following the regulation schedule are shown with full lines. A few days in 2017 overall have either a lower or a higher energy content than any day in 2016. It means that the energy constraints are violated, as the schedule, based on the 2016 values, did not allocate enough power to correct the SOC. The dashed lines in Fig 11 , shows the results for a fixed regulation capacity of $\pm 6.9 \mathrm{~kW}$ in all the 15 hours. In this case it is still possible to maintain the SOC to the same degree as when following the schedule but not the final value, as less correcting power is available in the last hour.

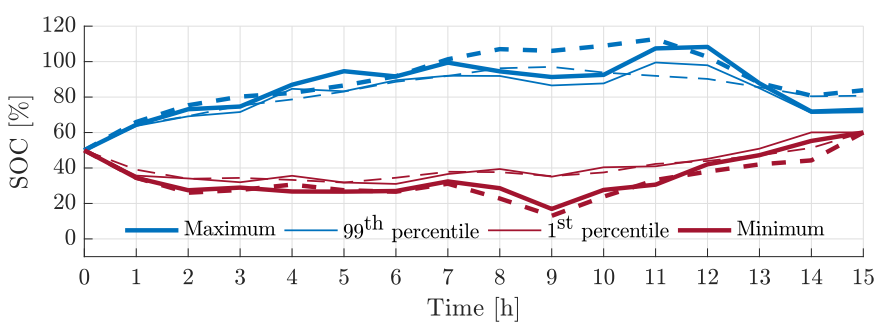

Fig. 11. The minimum, maximum, $1^{\text {st }}$ and $99^{\text {th }}$ percentiles of the realized SOCs for every scenario in 2017 . Full lines are for $P_{h}^{\mathrm{r}}$ equal to the schedule and the dashed lines are for $P_{h}^{\mathrm{r}}=6.9 \mathrm{~kW}$. The 15 hour periods take place daily at hour 16:00-07:00 


\section{Economic results}

Providing $\pm 10 \mathrm{~kW}$ of FNR daily between 16.00-07:00 with the average prices of 2017 would give a revenue of 1395 EUR per year. By bidding all of the power capacity, and not leaving any power to correct losses and the energy content, $88 \%$ of the scenarios will in the end of the period have an SOC outside the range of the battery capacity. Such a strategy would lead to SOC violations which would require either set point adjustments or it would lead to failure in service delivery. Nonetheless it would lead to the maximum potential earnings from providing FNR with the full capacity, and is consistent with the results from [7].

In the unidirectional case with the service provided during the hours with the highest prices (from midnight and the following four hours), and a capacity of $\pm 1.15 \mathrm{~kW}$, the average prices in 2016 would give a yearly revenue of 70 EUR per EV. Three daily hours would give a revenue of 52 EUR per EV. Four hours of unidirectional FNR service per day is the best case, whereas three hours are more realistic, for this driving behaviour. The value of bidirectional FNR with the ENEL chargers is about 20 times higher than FNR from unidirectional charging, because of the low availability time and power range, as shown in table II.

TABLE II

ECONOMIC COMPARISON OF FNR PROVISION METHODS

\begin{tabular}{c|c|c|c|c|c} 
Method & $\begin{array}{c}\text { Provision } \\
\text { Time } \\
{[\text { hours] }}\end{array}$ & $\begin{array}{c}\text { FNR } \\
\text { Capacity } \\
{[\mathrm{kW}]}\end{array}$ & $\begin{array}{c}\text { Yearly } \\
\text { Revenue } \\
{[\text { [EUR] }}\end{array}$ & $\begin{array}{c}\text { Yearly } \\
\text { Cost } \\
{[\text { EUR] }}\end{array}$ & $\begin{array}{c}\text { Yearly } \\
\text { Profit } \\
{[\text { [EUR] }}\end{array}$ \\
\hline V2G & 15 hours & \pm 10 & 1395 & 297 & 1097 \\
V2G & 15 hours & Schedule & 1118 & 208 & 910 \\
V2G & 15 hours & \pm 6.9 & 1107 & 208 & 899 \\
Uni & 4 hours & \pm 1.15 & 70 & - & 70 \\
Uni & 3 hours & \pm 1.15 & 52 & - & 52
\end{tabular}

Bidding FNR according to the optimised schedule gives a capacity payment of 1118 EUR per year, and a consumption for both FNR and driving of $6.7 \mathrm{MWh}$, with a cost of of 538 EUR per year. The $9 \mathrm{kWh}$ of daily SOC increases and the associated losses amounts to, amounts to $4.1 \mathrm{MWh}$ per year with a cost of 331 EUR. The energy for driving should be subtracted from the energy consumption for the service provision, giving a service cost of 208 EUR per year.

This describes the full value created by the EV and should be split between the aggregator and the EV owner. The method used in the ACES project is that the municipality gets free electricity for driving, while the aggregator can keep the remaining profit, which is most of the revenue as the aggregator only pays the industrial electricity price. In that case the aggregators share is a yearly profit of 580 EUR per EV.

The average regulation power capacity over the 15 hours is $\pm 7 \mathrm{~kW}$, leaving $\pm 3 \mathrm{~kW}$ for correcting the SOC. A fixed schedule with the same $\pm 6.9 \mathrm{~kW}$ regulation capacity in every hour would give a capacity payment of 1107 EUR per year, close to the payment of the calculated schedule. Using a fixed regulation capacity achieves the same results in terms of respecting the energy constraints as the schedule when used with the frequency from 2017.
The revenue is calculated with the historical prices and would increase linearly if the prices go up in the future. This could be the case as the demand for regulating power increases when the power system is based on a larger share of renewable energy. On the other hand, an increasing number of units delivering the service could also have the opposite effect on the prices. The Danish TSO is nowadays procuring $50 \mathrm{MW}$ of PFC and the considered set of EV and V2G charger can deliver $\pm 6.9 \mathrm{~kW}$, which means that the national demand can be satisfied with only 7246 EVs. It is worth noting that even though the amount of reserve will increase with the increase of renewable generation, it has to be compared with the total number of vehicles in Denmark, which is 2.3 million.

\section{Value of not fully charging the EVs}

Knowing that only a small part of the battery capacity is used for the daily driving requirements, it is possible to provide FNR for more time, using this flexibility. In Table [IV] it is shown how much the minimum SOC at the end of each period affects the yearly capacity payment. The increased payment is caused by both longer regulation time and increased average power capacity, $\overline{P_{h}^{\mathrm{r}}}$.

TABLE III

YEARLY CAPACITY PAYMENT AND AVERAGE FNR CAPACITY FOR DIFFERENT MINIMUM SOC AT THE END OF PROVISION

\begin{tabular}{c|c|c|c|c|c|c} 
End SOC & $\geq 40 \%$ & $\geq 50 \%$ & $\geq 60 \%$ & $\geq 70 \%$ & $\geq 80 \%$ & $=90 \%$ \\
\hline$\overline{P_{h}^{\mathrm{r}}}[ \pm \mathrm{kW}]$ & 7.7 & 7.4 & 7.2 & 7.0 & 6.7 & 6.5 \\
\hline EUR & 1239 & 1200 & 1160 & 1117 & 1072 & 1025
\end{tabular}

The optimisation is also run on a seasonal basis with only 91 scenarios, which shows the that since there are more energy content in the winter, the regulation capacity must be reduced and therefore also the earnings. The same average prices were used for all the seasons, but as mentioned in the analysis section, the prices are on average also higher in the summer.

TABLE IV

SEASONAL CAPACITY PAYMENT AND AVERAGE FNR CAPACITY FOR DIFFERENT SEASONS

\begin{tabular}{c|c|c|c|c} 
Season & Winter & Spring & Summer & Autum \\
\hline$\overline{P_{h}^{\mathrm{r}}}[ \pm \mathrm{kW}]$ & 7.2 & 7.0 & 7.5 & 7.0 \\
\hline EUR & 288 & 278 & 307 & 283
\end{tabular}

The energy losses related to providing the service are on an average day $7 \mathrm{kWh}$, which involves a large cost, even considering the very low industrial electricity price, equal to $0.08 \mathrm{EUR} / \mathrm{kWh}$. This would exceed the aggregator's revenue if he would pay the domestic electricity price. However, these chargers are on a prototype level, which means that while the revenue will not change, the cost for energy losses can be expected to decrease significantly in the future, when more mature products enter the market. The cost is only including the cost of electricity and not the cost of installation and maintenance, as well as the cost of additional wear of the battery, which is discussed in section IV-E 


\section{E. Considerations on battery degradation}

One of the aspects without a clear consensus within the $\mathrm{V} 2 \mathrm{G}$ research area is the cost of using the battery in terms of added battery degradation costs. The factors influencing battery degradation, measured as capacity fade, can be divided in two; calendar ageing which is a function of time and cycle ageing which is a function of the number of charge/discharge cycles. Most of the capacity fade is caused by calendar ageing depending on the SOC and temperature during storage, which means that it is far more important how the battery is treated while idle than how it is used [29].

Especially for EV applications, calendar ageing tends to be the dominating effect; this boils down to the fact that battery degradation is mostly time and temperature dependent, however economic cost calculations to date have mostly focused on cycle number as the determining factor of lifetime [30]. Ref. [31] experimentally finds that depending on the temperature, there is a $2-3 \%$ capacity loss per 1000 full equivalent cycles.

Ref. [32] finds that degradation is more influenced from calendar ageing and the battery degradation is twice as much if it is stored fully charged than stored at a SOC lower than $60 \%$. To maximise battery life, lithium-ion cells should not be stored at high SOC and for long-term storage, the SOC should be less than $50 \%$ [33]. It therefore has a positive effect on the state of health that the EV is not fully charged at the end of the day and stored fully charged all night.

In [32] it is experimentally shown that the battery degradation cannot only be based on the energy throughput as it depends on the absolute movement of the SOC, meaning the depth of discharge. It is found by [32] that by cycling the battery between $40-60 \%$, it had lost $14 \%$ of the original capacity after 3000 full equivalent cycles with a charge rate of $1 \mathrm{C}$ and a temperature of $35^{\circ} \mathrm{C}$. By integrating the absolute energy flow of the battery the total energy throughput, $E_{\mathrm{tp}}$, can be calculated with Eq. 14 The daily energy used for driving, $E_{\text {drive }}$, is subtracted from the total energy throughput, to get the added throughput from FNR provision.

$$
E_{\mathrm{tp}}=\sum_{k=1}^{365} \sum_{h=1}^{15} \Delta T\left(P_{k, h}^{\mathrm{c}} \eta_{\mathrm{c}}+\left|e_{k, h}^{\mathrm{bat}} P_{h}^{\mathrm{r}}\right|+P_{k, h}^{\mathrm{d}} \frac{1}{\eta_{\mathrm{d}}}\right)-E_{\text {drive }, k}
$$

Following the optimal schedule the battery has a yearly energy throughput of $E_{\mathrm{tp}}=12.2 \mathrm{MWh}$ per year. This is calculated by integrating the absolute energy flow of each hour of every period in the year, and subtracting the energy used for driving, $E_{\text {drive }}$. With a $40 \mathrm{kWh}$ battery it results in $E_{\text {tp }} /(2 Q)=153$ charge cycles per year.

The energy throughput calculated on the hourly scale is lower than if it is calculated on the second scale based on the full resolution time series, as some of the power flow cancels out within the hour. The yearly energy throughput calculated on the second scale is, however not significantly higher and amounts to $E_{\mathrm{tp}}=14.6 \mathrm{MWh}$ or 182 charge cycles per year.

In collaboration with Nissan, the authors in [34], have modelled the battery degradation of the same setup as presented in the present manuscript and the result is $0.4 \%$ capacity fade per year caused by service provision. Based on a single balanced frequency sample, that is repeated every day, ref. [34] also finds the throughput in the second scale to be equal to 182 full equivalent charge cycles per year. Despite delivering 14 hours of PFC with $\pm 9 \mathrm{~kW}$ per day, the degradation is not found to be a significant factor as the bulk discharge is limited and the cycle ageing only is a small part of the overall degradation.

The optimal schedule deals with several days of very imbalanced frequency but still results in the same average number of charge cycles, and therefore should also result in $0.4 \%$ cycle degradation per year. This should be compared with the magnitude of the calendar ageing, which is $2.8 \%$ in the first year and then close to $1 \%$ the following years [34]. These values are similar to the results from Ref. [35]. Most of the capacity fade of the battery is caused by calendar ageing which is considered independent of the number of cycles. The revenue values previously obtained are therefore expected to be marginally affected by degradation, though further experimental investigations are necessary to confirm this.

\section{CONCLUSION AND FUTURE WORK}

The paper first introduced the regulatory framework and potential earnings from frequency regulation in RG-N, based on the technical capabilities of series produced EVs in different configurations.

It is found that the value of unidirectional FNR during charging only is up to 70 EUR per year per EV, and while this method does not give any further energy loss as the EV always is charging, it gives a very limited revenue. Using bidirectional V2G chargers the value of the full power availability of \pm 10 $\mathrm{kW}$ is 1395 EUR per year per vehicle although it has the need for extra equipment.

It is found that the behaviour of the system frequency would cause the EV to be fully charged or depleted in $88 \%$ of the days in a year, if the full power is committed to the service. By assuming full knowledge of the energy content of the frequency, it is possible to make a schedule that would not fully charge or deplete the battery. This schedule has been calculated based on the frequency values of 2016 and applied to the days of 2017. In 2016 it would generate a capacity payment of 1118 EUR per year per vehicle with an average regulation capacity of $\pm 6.9 \mathrm{~kW}$ out of a nominal capacity of $\pm 10 \mathrm{~kW}$. This amount represents the best-case earnings that can be achieved given the adopted hardware. It is suggested, that the aggregator remunerates the $\mathrm{EV}$ owner by paying for the electricity consumption for driving, in which case the aggregators share of the profit is $580 \mathrm{EUR} / \mathrm{year}$. The EV owner needs a proper remuneration for the use of the battery and for only having the EV $72.5 \%$ charged in the morning, even though a lower SOC have a positive effect on the calendar ageing of the battery.

The experimental results of the commercial V2G charger shows that the energy losses due to the charger efficiency amounts to $4.2 \mathrm{MWh}$ out of a total energy throughput of the battery of $18.8 \mathrm{MWh}$ per year, including driving. 
Even considering the reduced industrial electricity tariff, it determines a loss in revenue close to one fifth. Considering the collected experience on battery degradation, the influence of frequency regulation in the given setup is found to have marginal consequences. Though further practical investigations are intended to back up the statement.

As final consideration, it is found that overall the business model is better in the summer as the prices are $30 \%$ higher, the energy content is lower so $8 \%$ more regulation capacity can be delivered and the energy requirements for driving are also lower so the minimum SOC could be lower the next morning.

While the paper quantified the value of each individual pair of EV and charger, it has to be remarked how the value, the energy costs and the number of stakeholders increase linearly with the number of EVs. Aggregating a large fleet can make it possible to predict the average SOC and align the individual EVs SOC but the revenue per EV will not be higher than the best case presented here.

\section{REFERENCES}

[1] S. Bashash and H. K. Fathy, "Cost-optimal charging of plug-in hybrid electric vehicles under time-varying electricity price signals," IEEE Transactions on Intelligent Transportation Systems, vol. 15, no. 5, Oct 2014.

[2] A. Schuller, C. M. Flath, and S. Gottwalt, "Quantifying load flexibility of electric vehicles for renewable energy integration," Applied Energy, vol. 151, pp. 335 344,2015

[3] M. Marinelli, S. Martinenas, K. Knezović, and P. Andersen, "Validating a centralized approach to primary frequency control with series-produced electric vehicles," Journal of Energy Storage, vol. 7, pp. 63 - 73, 2016.

[4] Y. V. Makarov, S. Lu, J. Ma, and T. B. Nguyen, "Assessing the value of regulation resources based on their time response characteristics," California Energy Commission, Public Interest Energy Research Program, Jun 2008.

[5] W. Kempton and J. Tomić, "Vehicle-to-grid power fundamentals: Calculating capacity and net revenue," Journal of Power Sources, vol. 144, no. 1, pp. 268 $-279,2005$.

[6] K. Knezović, M. Marinelli, A. Zecchino, P. B. Andersen, and C. Traeholt, "Supporting involvement of electric vehicles in distribution grids: Lowering the barriers for a proactive integration," Energy, vol. 134, pp. 458 - 468, 2017.

[7] A. Thingvad, S. Martinenas, P. Andersen, M. Marinelli, B. Christensen, and O. Olesen, "Economic comparison of electric vehicles performing unidirectional and bidirectional frequency control in denmark with practical validation," 2016 Proceedings of the 51st International Universities Power Engineering Conference, 2016.

[8] J. Hu, H. Morais, T. Sousa, and M. Lind, "Electric vehicle fleet management in smart grids: A review of services, optimization and control aspects," Renewable \& Sustainable Energy Reviews, vol. 56, p. 1207-1226, 2016.

[9] H. Zarkoob, S. Keshav, and C. Rosenberg, "Optimal contracts for providing load-side frequency regulation service using fleets of electric vehicles," Journal of Power Sources, vol. 241, pp. 94 - 111, 2013.

[10] A. Kieldsen, A. Thingvad, S. Martinenas, and T. M. Sørensen, "Efficiency test method for electric vehicle chargers," Proceedings of EVS29-International Battery, Hybrid and Fuel Cell Electric Vehicle Symposium, 2016.

[11] A. Thingvad, C. Ziras, J. Hu, and M. Marinelli, "Assessing the energy content of system frequency and electric vehicle charging efficiency for ancillary service provision," Proceedings of the 51st International Universities Power Engineering Conference, 2017.

[12] Energinet.dk, "Ancillary services to be delivered in denmark - tender conditions." http://energinet.dk/EN/El/Systemydelser-for-el/Sider/Systemydelserforel.aspx. 2012.

[13] P. B. Andersen, T. Sousa, A. Thingvad, L. S. Berthou, and M. Kulahci, "Added Value of Individual Flexibility Profiles of Electric Vehicle Users For Ancillary Services." Aalborg: IEEE International Conference on Communications, Control, and Computing Technologies for Smart Grids, 2018.

[14] M. Pertl, F. Carducci, M. D. Tabone, M. Marinelli, S. Kiliccote, and E. C. Kara, "An equivalent time-variant storage model to harness ev flexibility: Forecast and aggregation," IEEE Transactions on Industrial Informatics, pp. 1-1, 2018.

[15] N. Rotering and M. Ilic, "Optimal charge control of plug-in hybrid electric vehicles in deregulated electricity markets," IEEE Transactions on Power Systems, vol. 26, no. 3, pp. 1021-1029, 2011.

[16] E. Yao, V. W. S. Wong, and R. Schober, "Robust Frequency Regulation Capacity Scheduling Algorithm for Electric Vehicles," vol. 8, no. 2, 2017.

[17] J. Engels, B. Claessens, and G. Deconinck, "Combined stochastic optimization of frequency control and self-consumption with a battery," IEEE Transactions on Smart Grid, pp. 1-1, 2017.

[18] J. Fleer and P. Stenzel, "Impact analysis of different operation strategies for battery energy storage systems providing primary control reserve," Journal of Energy Storage, vol. 8, pp. $320-338,2016$.
[19] Y. Wang, C. Wan, Z. Zhou, K. Zhang, and A. Botterud, "Improving deployment availability of energy storage with data-driven agc signal models," IEEE Transactions on Power Systems, vol. PP, pp. 1-1, 122017.

[20] J. Donadee and J. Wang, "Agc signal modeling for energy storage operations," IEEE Transactions on Power Systems, vol. 29, no. 5, pp. 2567-2568, Sept 2014.

[21] S. I. Vagropoulos and A. G. Bakirtzis, "Optimal bidding strategy for electric vehicle aggregators in electricity markets," IEEE Transactions on Power Systems, vol. 28, no. 4, pp. 4031-4041, Nov 2013.

[22] Y. A. Shirazi and D. L. Sachs, "Comments on "measurement of power loss during electric vehicle charging and discharging" - notable findings for v2g economics," Energy, 2017.

[23] N. B. Arias, S. Hashemi, P. B. Andersen, C. Traholt, and R. Romero, "V2G enabled EVs providing frequency containment reserves: Field results," Proceedings of the IEEE International Conference on Industrial Technology, pp. 1814-1819, 2018.

[24] M. Trahand, "Nuvve v2g \& deployments, presented at the 2nd vehicle 2 grid conference, electric vehicles for the renewable city, amsterdam university of applied sciences," http://amsterdamv2gconference.eu/images/program/NUVVE\% 20-\%20V2G\%20Conference17.pdf 2017.

[25] EUROSTAT, "Electricity price statistics," http://ec.europa.eu/eurostat/ statistics-explained/index.php/Electricity_price_statistics\#Electricity_prices_ for non-household consumers 2018.

[26] ACES, "Project website," www.aces-bornholm.eu 2016.

[27] J. Lofberg, "Yalmip : a toolbox for modeling and optimization in matlab," in 2004 IEEE International Conference on Robotics and Automation (IEEE Cat. No.04CH37508), Sept 2004, pp. 284-289.

[28] C. Ziras, A. Zecchino, and M. Marinelli, "Response accuracy and tracking errors with decentralized control of commercial v2g chargers," in 2018 Power Systems Computation Conference (PSCC), June 2018, pp. 1-7.

[29] D. Li, D. L. Danilov, J. Xie, L. Raijmakers, L. Gao, Y. Yang, and P. H. Notten, "Degradation Mechanisms of C6/LiFePO4Batteries: Experimental Analyses of Calendar Aging," Electrochimica Acta, vol. 190, pp. 1124-1133, 2016.

[30] A. W. Thompson, "Economic implications of lithium ion battery degradation for vehicle-to-grid (v2x) services," Journal of Power Sources, vol. 396, pp. 691 - 709, 2018

[31] J. Wang, J. Purewal, P. Liu, J. Hicks-Garner, S. Soukazian, E. Sherman, A. Sorenson, L. Vu, H. Tataria, and M. W. Verbrugge, "Degradation of lithium ion batteries employing graphite negatives and nickel-cobalt-manganese oxide + spinel manganese oxide positives: Part 1, aging mechanisms and life estimation," Journal of Power Sources, vol. 269, pp. 937 - 948, 2014.

[32] M. Ecker, N. Nieto, S. Käbitz, J. Schmalstieg, H. Blanke, A. Warnecke, and D. U. Sauer, "Calendar and cycle life study of li(nimnco)o2-based 18650 lithium-ion batteries," Journal of Power Sources, vol. 248, pp. 839 - 851, 2014.

[33] P. Keil, S. Schuster, J. Wilhelm, J. Travi, A. Hauser, R. C. Karl, and A. Jossen, "Calendar aging of lithium-ion batteries: I. impact of the graphite anode on capacity fade," Journal of The Electrochemical Society, vol. 163, pp. A1872-A1880, 01 2016.

[34] A. Thingvad and M. Marinelli, "Influence of v2g frequency services and driving on electric vehicles battery degradation in the nordic countries," in EVS31, 2018.

[35] K. Darcovich, S. Recoskie, H. Ribberink, F. Pincet, and A. Foissac, "Effect on battery life of vehicle-to-home electric power provision under canadian residential electrical demand," Applied Thermal Engineering, vol. 114, pp. 1515 - 1522, 2017. 